\title{
Overexpression of TNF- $\alpha$-converting enzyme in fibroblasts augments dermal fibrosis after inflammation
}

\author{
Shinji Fukaya ${ }^{1,2,}$, Yuki Matsui ${ }^{3, *}$, Utano Tomaru ${ }^{1}$, Ai Kawakami ${ }^{3}$, Sayuri Sogo ${ }^{4}$, Toshiyuki Bohgaki ${ }^{2}$, \\ Tatsuya Atsumi ${ }^{2}$, Takao Koike ${ }^{2}$, Masanori Kasahara ${ }^{1}$ and Akihiro Ishizu ${ }^{5}$
}

TNF- $\alpha$-converting enzyme (TACE) can cleave transmembrane proteins, such as TNF- $\alpha$, TNF receptors, and epidermal growth factor receptor (EGFR) ligands, to release the extracellular domains from the cell surface. Recent studies have suggested that overexpression of TACE may be associated with the pathogenesis of inflammation and fibrosis. To determine the roles of TACE in inflammation and fibrosis, TACE transgenic (TACE-Tg) mice, which overexpressed TACE systemically, were generated. As the transgene-derived TACE was expressed as an inactive form, no spontaneous phenotype developed in TACE-Tg mice. However, the transgene-derived TACE could be converted to an active form by furin in vitro and by phorbol myristate acetate (PMA) in vivo. Subcutaneous injection of PMA into mice induced inflammatory cell infiltration 1 day later and subsequent dermal fibrosis 7 days later. Interestingly, the degree of dermal fibrosis at day 7 was significantly higher in TACE-Tg mice than in wild-type mice. Correspondingly, PMA increased the expression of type I collagen in the primary culture of dermal fibroblasts derived from TACE-Tg mice. Furthermore, phosphorylated EGFR was increased in the fibroblasts by the PMA treatment. The collective findings suggest that TACE overexpression and activation in fibroblasts could shed off putative EGFR ligands. Subsequently, the soluble EGFR ligands could bind and activate EGFR on fibroblasts, and then increase the type I collagen expression resulting in induction of dermal fibrosis. These results also suggest that TACE and EGFR on fibroblasts may be novel therapeutic targets of dermal fibrosis, which is induced after diverse inflammatory disorders of the skin.

Laboratory Investigation (2013) 93, 72-80; doi:10.1038/labinvest.2012.153; published online 12 November 2012

KEYWORDS: EGFR; fibrosis; inflammation; PMA; TACE

TNF- $\alpha$-converting enzyme (TACE), which belongs to a disintegrin and metalloproteinase (ADAM) family, can cleave transmembrane proteins to release the extracellular domains from the cell surface. ${ }^{1,2}$ Initially produced as an inactive $120 \mathrm{kDa}$ protein, the $\mathrm{N}$-terminus prodomain is removed by furin at the trans-golgi network, and consequently TACE is converted to an active form of $100 \mathrm{kDa}$ protein. ${ }^{3-6}$ The active form of TACE is transported to the plasma membrane and binds to its substrates on the cell surface. Substrates of TACE include TNF- $\alpha$, TNF receptors, and epidermal growth factor receptor (EGFR) ligands.

When focusing on the role of TNF- $\alpha$ in inflammation, it is considered that TACE contributes to promote inflammation by increasing soluble TNF- $\alpha$. However, it is also considered that TACE plays a role in the suppression of inflammation by decreasing membrane-type TNF receptors and producing soluble TNF receptors, which can work as decoy receptors. These concepts seem contradictory, but TACE really functions to maintain the physiological homeostasis. The expression of TACE substrates is strictly regulated in a timedependent manner during the inflammation process.

On the other hand, it has been demonstrated that rat collagen antibody-induced arthritis and lipopolysaccharide (LPS)-induced acute lung injury can be treated by TACE inhibitors. ${ }^{7,8}$ Recently, Terao et al ${ }^{9}$ have demonstrated that murine bleomycin-induced scleroderma could also be treated by TACE inhibitors. These findings suggest that TACE may be critically involved in the pathogenesis of these inflammatory

\footnotetext{
${ }^{1}$ Department of Pathology, Hokkaido University Graduate School of Medicine, Sapporo, Japan; ${ }^{2}$ Department of Internal Medicine II, Hokkaido University Graduate School of Medicine, Sapporo, Japan; ${ }^{3}$ Graduate School of Health Sciences, Hokkaido University, Sapporo, Japan; ${ }^{4}$ School of Health Sciences, Hokkaido University, Sapporo, Japan and ${ }^{5}$ Faculty of Health Sciences, Hokkaido University, Sapporo, Japan

Correspondence: Professor A Ishizu, MD, PhD, Faculty of Health Sciences, Hokkaido University Kita-12, Nishi-5, Kita-ku, Sapporo Hokkaido 060-0812, Japan. E-mail: aishizu@med.hokudai.ac.jp

*These authors contributed equally to this work.

Received 17 January 2012; revised 19 September 2012; accepted 25 September 2012
} 
and fibrous connective tissue diseases. However, the precise mechanism of the implication of TACE in inflammation and fibrosis has not been revealed. This study aimed to clarify the role of TACE in the pathogenesis of inflammation and fibrosis using TACE transgenic (TACE-Tg) mice, which could overexpress TACE in the systemic organs.

\section{MATERIALS AND METHODS}

\section{Generation of TACE-Tg Mice}

The transgene for generation of TACE-Tg mice contained the full-length mouse TACE cDNA, which connected the Flag tag to the $3^{\prime}$ region. The connection of the Flag tag rendered distinction of the transgene-derived TACE from the endogenous TACE. The construct was inserted into pCAGGS vector containing the $\beta$-actin promoter, which could bring systemic expression of the transgene. Then, the pCAGGS vector carrying the transgene was microinjected into fertilized eggs of BDF1 mice at Genome Information Research Center, Research Institute of Microbial Disease, Osaka University (Osaka, Japan). Four founder mice obtained were mated with C57BL/6 mice (Japan Clea, Tokyo, Japan), and then the offspring mice were backcrossed into C57BL/6 mice more than 6 times. Among them, one stable line of TACE-Tg mice with heterozygous transgene insertion was served for this study. Age-matched wild-type (WT) C57BL/6 mice were used for the control. Experiments using mice were done in accordance with the guidelines for the care and use of laboratory animals in Hokkaido University.

\section{Real-Time RT-PCR}

For RNA extraction from mouse tissues, RNeasy Mini kit (Qiagen, Hilden, Germany) was used. After digestion of contaminated genomic DNA by DNase I, RNA was reverse transcribed to cDNA using Superscript III First-Strand Synthesis System (Invitrogen, Carlsbad, CA, USA). The expression of TACE mRNA was quantified by real-time RT-PCR using QuantiTect SYBR Green PCR kit (Qiagen). The primer sequences for TACE were as follows: $5^{\prime}$-ATCTG AAGAGTTTGTTCGTCGAG-3' (sense) and $5^{\prime}$-TCCACGG CCCATGTATTTAT-3' (antisense). PCR was run on ABI Prism 7000 (Applied Biosystems, Carlsbad, CA, USA) as follows: after denaturation at $95^{\circ} \mathrm{C}$ for $10 \mathrm{~min}, 40$ cycles of reaction at $95^{\circ} \mathrm{C}$ for $15 \mathrm{~s}$ and at $56^{\circ} \mathrm{C}$ for $60 \mathrm{~s}$ were carried out. For the internal control, the expression of hypoxanthineguanine phosphoribosyltransferase 1 (HPRT1) was monitored. The primer sequences for HPRT-1 were as follows: $5^{\prime}$-TGGAAAGAATGTCTTGATTGTTGAA- $3^{\prime}$ (sense) and 5'-AGCTTGCAACCTTAACCATTTTG-3' (antisense).

\section{Western Blotting}

The mouse tissues were homogenized in lysis buffer $(0.1 \%$ sodium dodecyl sulfate (SDS), $1 \%$ Nonidet-P40, $0.5 \%$ sodium deoxycholate, $100 \mu \mathrm{g} / \mathrm{ml}$ phenylmethylsulfonyl fluoride, $1 \mathrm{mM}$ sodium orthovanadate, protease inhibitor cocktail (Complete Mini, Roche, Basel, Switzerland)). The lysates adjusted ranging from 10 to $40 \mu \mathrm{g} /$ lane were fractionated on $7.5 \%$ SDS polyacrylamide gel and then transferred onto PVDF membranes (GE Healthcare, Buckinghamshire, UK). After blocking by TBS-T (0.1\% Tween-20 in Tris-buffered saline) containing 2\% non-fat milk, the membranes were incubated with 1:5000 dilution of the anti-TACE antibody (Santa Cruz Biotechnology, Santa Cruz, CA, USA) or 1:5000 dilution of the anti-Flag antibody (Sigma-Aldrich, St Louis, MO, USA) overnight at $4{ }^{\circ} \mathrm{C}$. After 3 times of wash by TBS-T, the membranes were next incubated with 1:25000 dilution of the peroxidase-labeled secondary antibodies (GE Healthcare) overnight at $4{ }^{\circ} \mathrm{C}$. Protein bands were detected using ECL Advance Western Blotting Detection kit (GE Healthcare). The anti-TACE antibody used in this study could react with the C-terminus of mouse TACE; thus, it could detect both the inactive and active forms.

\section{Activation of TACE by Furin In Vitro}

The skin tissues obtained from TACE-Tg and WT mice were lysed in the furin assay buffer (100 mM HEPES ( $\mathrm{pH} 7.5)$, $0.5 \%$ Triton $\mathrm{X}-100,1 \mathrm{mM} \mathrm{CaCl}_{2}$, and $1 \mathrm{mM}$ 2-mercaptoethanol). The lysates $(140 \mu \mathrm{g} / 100 \mu \mathrm{l})$ were incubated with recombinant human furin (Sigma-Aldrich) at respective concentrations of $0,0.1$, and $1 \mathrm{unit} / \mu \mathrm{l}$ for $1 \mathrm{~h}$ at $30^{\circ} \mathrm{C}$. The samples were then fractionated on $7.5 \%$ SDS polyacrylamide gel, and western blotting was performed using the anti-TACE or anti-Flag antibodies.

\section{Measurement of TACE Activity}

The skin lysates treated by furin were subjected to measurement of TACE activity. The TACE activity was measured using SensoLyte 520 TACE Activity Assay kit (AnaSpec, Fremont, CA, USA) and Varioskan Flash Microplate Multimode Readers (Thermo Fisher Scientific, Waltham, MA, USA) according to the manufacturer's protocol.

\section{Primary Culture of Dermal Fibroblasts}

The back skin of TACE-Tg and WT mice were turned inside out and $3 \mathrm{~mm}$ pieces of the dermis were excised, put on flat dishes, and then incubated in RPMI-1640 (Sigma-Aldrich) containing $20 \%$ fetal calf serum, $50 \mu \mathrm{g} / \mathrm{ml}$ streptomycin, and $50 \mathrm{U} / \mathrm{ml}$ penicillin. Several days later, spindle-shaped cells migrated and proliferated around the skin pieces. After removal of the skin pieces, the cells were used as primary culture of dermal fibroblasts. Experiments were conducted using the cells at $3-5$ passages.

\section{Stimulation of Dermal Fibroblasts by PMA}

The primary culture of dermal fibroblasts was stimulated by phorbol myristate acetate (PMA; LC Laboratories, Woburn, MA, USA) at respective concentrations of $0,6.4,64$, and $640 \mathrm{nM}$. After $30 \mathrm{~min}$ of incubation at $37^{\circ} \mathrm{C}$, the cells were lysed, and then the lysates were served for the anti-TACE or anti-Flag immunoblotting. 


\section{Subcutaneous Injection of PMA}

TACE-Tg and WT mice (10 weeks old, female) with shaved back skin were subcutaneously injected with $0.08 \mu \mathrm{g}$ PMA in $0.1 \mathrm{ml}$ PBS (1300 nM) using $29 \mathrm{G}$ syringe needle. As control, the same volume of PBS without PMA was injected subcutaneously.

\section{Histological Evaluation}

At 1 and 7 days after the inoculation, the skin sites with PMA and PBS injections were excised as $6 \mathrm{~mm}$ round-shaped pieces, fixed in formalin, and then subjected to hematoxylin and eosin (HE) staining. The samples at 7 days were also subjected to Elastica-Masson (EM) staining. Dermal thickening ratio was calculated as follows: (1) thickness of dermis was measured at three random points of the sites with PBS injection, (2) mean thickness at the sites with PBS injection was calculated, (3) thickness of dermis was measured at three random points of the sites with PMA injection, and (4) dermal thickness ratios were calculated by dividing the thickness of dermis at the PMA injection sites by the mean dermal thickness at the sites with PBS injection.

\section{Expression of Type I Collagen}

To evaluate fibrosis in the molecular level, the expression of type I collagen (collagen 1A1) was examined by real-time RTPCR. First, at 7 days, the skin sites with PMA and PBS injections were excised as $6 \mathrm{~mm}$ round-shaped pieces, RNA was extracted from the tissues, and then real-time RT-PCR was performed as described above. The primers for collagen 1A1 were as follows: $5^{\prime}$-GAGCCCTCGCTTCCGTACTC-3' (sense) and 5'-TGTTCCCTACTCAGCCGTCTGT-3' (antisense). Next, the primary culture of dermal fibroblasts was treated by PMA at respective concentrations of $0,20,160$, and $1300 \mathrm{nM}$. After $4 \mathrm{~h}$ of incubation at $37^{\circ} \mathrm{C}$, RNA was extracted from the cells, and then the expression of collagen $1 \mathrm{~A} 1$ was examined similarly by real-time RT-PCR.

\section{TACE Inhibition Assay}

The fibroblasts derived from TACE-Tg mice were treated by $1300 \mathrm{nM}$ of PMA with or without $25 \mu \mathrm{g} / \mathrm{ml}$ of TAPI-0 (Enzo Life Sciences, Farmingdale, NY, USA) at $37^{\circ} \mathrm{C}$. TAPI-0 can inhibit TACE and other matrixmetalloproteases. ${ }^{10}$ The concentration of TAPI- 0 was adopted according to the literature. ${ }^{11}$ After $4 \mathrm{~h}$ of incubation, RNA was extracted from the cells, and then the expression of collagen $1 \mathrm{~A} 1$ was examined by real-time RT-PCR as described above.

\section{Detection of Phosphorylated EGFR}

To determine if EGFR was activated by PMA, phosphorylation of EGFR was examined. The primary culture of dermal fibroblasts was treated by PMA at respective concentrations of $0,20,160$, and $1300 \mathrm{nM}$. After $1 \mathrm{~h}$ of incubation at $37^{\circ} \mathrm{C}$, RNA and cell lysates were extracted. The RNA was then served for RT-PCR using the EGFR primers (sense: 5'GAACTGGGCTTAGGGAACTGC-3' , antisense: 5'-CATTGG
GACAGCTTGGATCAC-3'), and the lysates were served for western blotting using the anti-phosphorylated EGFR antibody (Phospho-EGF Receptor (Tyr1068); Cell Signaling Technology, Tokyo, Japan). RT-PCR was carried out as described above. As internal controls, the expression of HPRT1 and the amount of actin detected by the anti-actin antibody (Chemicon International, Temecula, CA, USA) were monitored.

\section{Statistics}

Data were presented as mean $\pm \mathrm{s}$ d. Student's $t$-test was applied for statistical analysis. The $P$-value of $<0.05$ was considered to be significant.

\section{RESULTS}

\section{Overexpression of TACE in TACE-Tg Mice}

The TACE mRNA and protein expressions in the systemic organs of 6-week-old TACE-Tg and WT mice were evaluated by real-time RT-PCR and western blotting, respectively. The TACE mRNA expression in all organs examined was higher in TACE-Tg mice than in WT mice, although the expression level was variable among organs (Figure 1a). The top five organs with the highest level of expression of TACE mRNA included the muscle, pancreas, heart, skin, and intestine. In WT mice, the TACE mRNA expression was relatively high in the pancreas and skin. The TACE protein expression, which was detected by the anti-TACE immunoblotting, well reflected the mRNA expression (Figure 1b). These findings
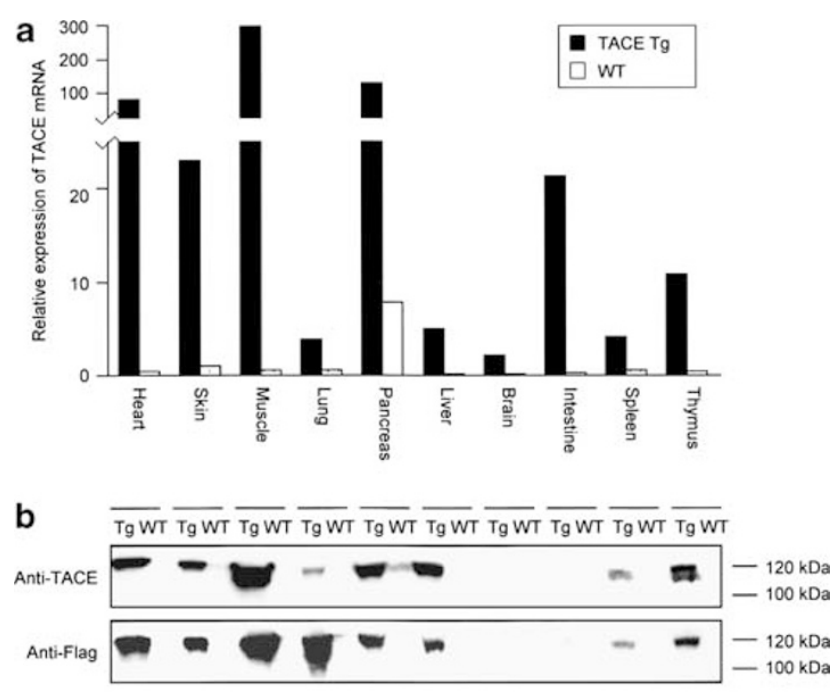

Figure 1 TACE mRNA and protein expressions in TACE-Tg and WT mice. The mRNA expressions of TACE in systemic organs of 6-week-old TACE$\mathrm{Tg}$ and WT mice were quantified using real-time RT-PCR (a). The expression level in each organ was standardized by the level in the skin of WT mice. The expression of TACE protein was examined by western blotting (b). Lysates of systemic organs from 6-week-old TACE-Tg and WT mice were subjected to anti-TACE and anti-Flag immunoblotting. Experiments were repeated 3 times, and similar results were reproduced. Representative data are shown. 
indicated the overexpression of TACE in TACE-Tg mice compared with WT mice. The anti-Flag immunoblotting suggested that the difference in the amount of TACE between TACE-Tg and WT mice was attributable to the expression of the transgene-derived TACE. Remarkably, most of the transgene-derived TACE protein was detected as the $120 \mathrm{kDa}$ inactive form.

\section{No Spontaneous Phenotype in TACE-Tg Mice}

Comparison of histology of systemic organs between 6-weekold TACE-Tg mice and WT mice revealed no remarkable difference (Supplementary Figure 1). The TACE-Tg mice kept for up to 2 years showed no spontaneous development of a specific phenotype. This might be consistent with the presence of most of all transgene-derived TACE protein as the inactive form in TACE-Tg mice.

\section{Activation of TACE by Furin}

ADAM family molecules, including TACE, undergo proteolysis to the active form by protein convertases. ${ }^{3,4}$ The inactive TACE of $120 \mathrm{kDa}$ protein is removed in the $\mathrm{N}$-terminus prodomain and then converted to the active form of $100 \mathrm{kDa}$ protein by furin in vivo. ${ }^{5,6}$ To determine the catalytic capacity of transgene-derived TACE, the tissue lysates of skin from TACE-Tg and WT mice were incubated with furin in vitro. The western blotting using the anti-TACE and anti-Flag antibodies revealed that the transgene-derived TACE protein could be converted to the $100 \mathrm{kDa}$ active form by furin in a dose-dependent manner (Figure 2a). Compatible with these findings, the TACE activity in the samples from TACE-Tg mice was increased by furin dose-dependently, and the increased TACE activities in the samples from TACE-Tg mice exhibited significantly higher levels than those in WT samples (Figure 2b).

\section{Activation of TACE by PMA In Vitro}

Administration of furin into mice is difficult because furin exclusively functions in the cytoplasm in vivo. In this study, alternative stimulation, which can convert TACE to the active form, was sought; hence, PMA was employed. When the primary culture of dermal fibroblasts was stimulated by PMA, the transgene-derived TACE protein was effectively converted to the active form (Figure 3a). Although the TACE activity in WT fibroblasts was significantly increased by PMA dose-dependently as well as that in the TACE-Tg fibroblasts, the increased amount of active TACE in WT samples seemed to remain at an undetectable level of the anti-TACE immunoblotting (Figure 3b).

\section{Activation of TACE by PMA In Vivo}

To determine that PMA could convert TACE to the active form in vivo, PMA was subcutaneously injected into TACE$\mathrm{Tg}$ and WT mice, and then skin samples were obtained 1 and 7 days after the inoculation. Western blotting using the antiTACE and anti-Flag antibodies revealed that the active TACE
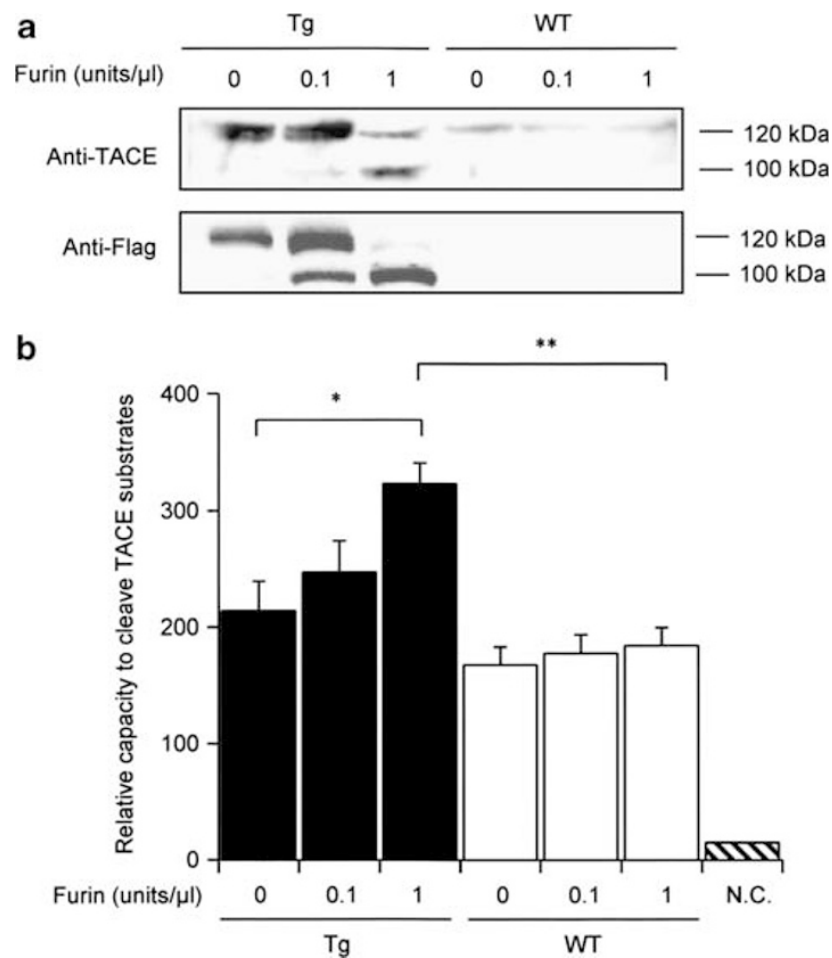

Figure 2 TACE activation by furin in vitro. Lysates of the skin from 6-week-old TACE-Tg and WT mice were incubated with furin at respective concentrations of $0,0.1$, and 1 unit/ $\mu \mathrm{l}$ for $1 \mathrm{~h}$ at $30^{\circ} \mathrm{C}$. The samples were then subjected to anti-TACE and anti-Flag immunoblotting (a). Experiments were repeated 3 times, and similar results were reproduced. Representative data are shown. TACE activities in the samples (TACE-Tg: $n=3$, WT: $n=3$ ) were measured using SensoLyte 520 TACE Activity Assay kit (b). NC represents the spontaneous cleavage of TACE substrates in the kit. ${ }^{*} P<0.05,{ }^{*} P<0.01$.

of $100 \mathrm{kDa}$ protein increased in the sites of PMA injection in TACE-Tg mice at day 1 . The amount of the active TACE in the sites of PMA injection in WT mice did not reach detection level (Figure 4a). The TACE activation recovered to the unstimulated level at 7 days after the PMA injection even in TACE-Tg mice. The TACE activity in the skin tissues of WT mice was significantly increased by PMA at day 1 as well as that in the TACE-Tg samples, although the increased amount of active TACE in the WT samples still remained at an undetectable level of the anti-TACE immunoblotting (Figure 4b).

\section{Augmented Dermal Fibrosis after PMA-Induced Inflammation in TACE-Tg Mice}

At 1 day after PMA injection, a severe infiltration of polymorphonuclear cells was observed in the subcutaneous tissue. Variety and degree of inflammatory cell infiltration were equivalent between TACE-Tg and WT mice (Figure 5). Thickening of the dermis and scar formation in the subcutaneous tissue were observed at the inflammation sites 7 days after the PMA injection (Figures 6a-h). The dermal thickening ratio (PMA injection site/PBS injection site) was significantly higher in TACE-Tg mice than in WT mice 
a

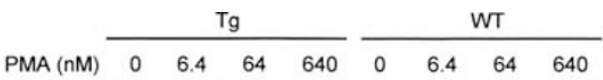

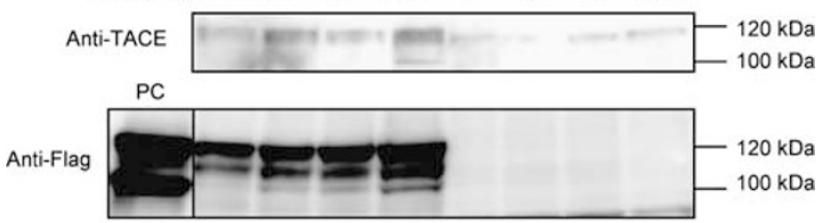

b

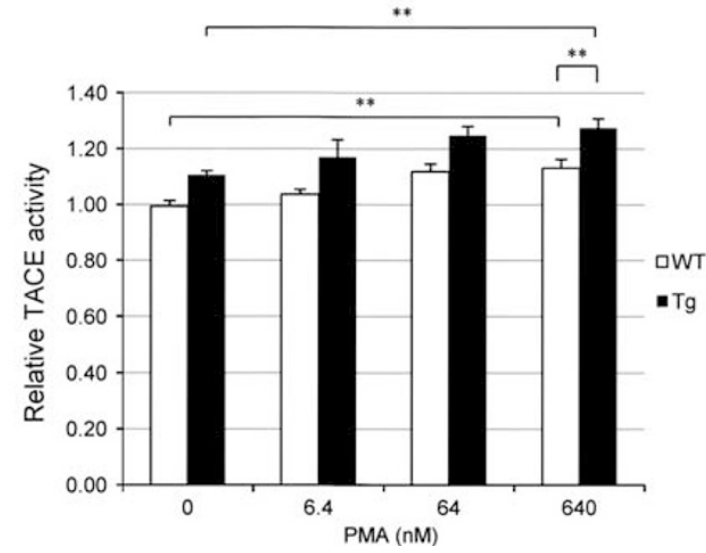

Figure 3 TACE activation by PMA in vitro. The primary culture of dermal fibroblasts derived from TACE-Tg and WT mice was stimulated by PMA at respective concentrations of $0,6.4,64$, and $640 \mathrm{nM}$. After $30 \mathrm{~min}$ of incubation at $37^{\circ} \mathrm{C}$, the cells were lysed, and then the lysates were subjected to anti-TACE and anti-Flag immunoblotting (a). Experiments were repeated 3 times, and similar results were reproduced.

Representative data are shown. PC: positive control (furin-treated TACE$\mathrm{Tg}$ skin lysates used in Figure 2). TACE activity in each sample $(n=3$, in each group) was measured using SensoLyte 520 TACE Activity Assay kit (b). ${ }^{*} P<0.01$.

(Figure 6i). Correspondingly, the mRNA expression of type I collagen in the skin 7 days after the PMA injection was relatively higher in TACE-Tg mice than in WT mice, though there was no statistical significant difference (Figure 6j). Notably, the type I collagen expression at the sites of PMA injection reached twofold level of the PBS-injected sites in TACE-Tg mice, whereas the expression at PMA injection sites was equal to that of the PBS injection sites in WT mice.

\section{Induction of Type I Collagen Expression in Dermal Fibroblasts by PMA}

To elucidate the hypothesis that the quantitative difference of TACE in dermal fibroblasts between TACE-Tg and WT mice was attributable to the degree of dermal fibrosis after the PMA-induced inflammation, the primary culture of dermal fibroblasts was stimulated by PMA in vitro, and then the mRNA expression of type I collagen was examined by realtime RT-PCR. As a result, the levels of type I collagen expression were upregulated by PMA dose-dependently in dermal fibroblasts of TACE-Tg mice, although the expression was not altered by the PMA treatment in dermal fibroblasts of WT mice (Figure 7a). In addition, the induction of type I collagen by PMA was significantly inhibited by the TACE inhibitor, TAPI-0 (Figure 7b). These findings suggested that
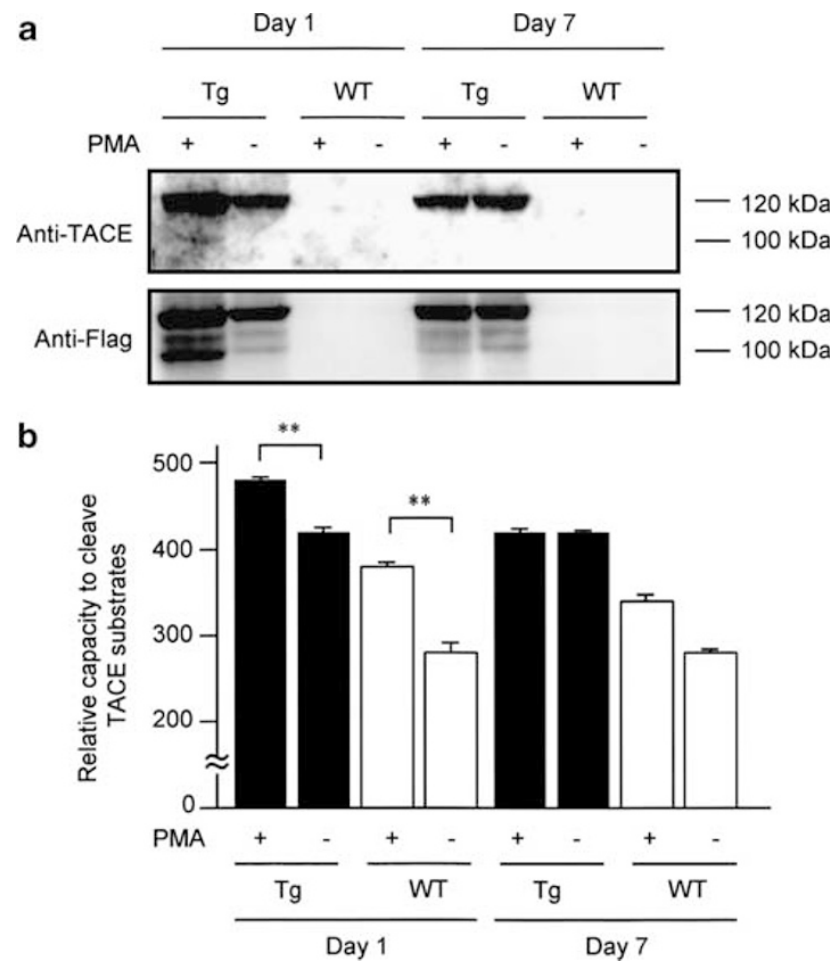

Figure 4 TACE activation by PMA in vivo. PMA $(0.08 \mu \mathrm{g} / 0.1 \mathrm{ml}$ PBS, $1300 \mathrm{nM}$ ) was injected subcutaneously into TACE-Tg and WT mice using $29 \mathrm{G}$ syringe needle. As a control, the same volume of PBS without PMA was injected subcutaneously. Lysates were obtained from the sites with PMA and PBS injection, respectively, at day 1 and day 7. Western blotting was performed using the anti-TACE and anti-Flag antibodies (a).

Experiments were repeated 3 times, and similar results were reproduced. Representative data are shown. TACE activity in each sample $(n=3$, in each group) was measured using SensoLyte 520 TACE Activity Assay kit (b). ${ }^{*} P<<0.01$.

the overexpression and activation of TACE in dermal fibroblasts could promote the type I collagen expression.

\section{Increase of Phosphorylated EGFR in Dermal Fibroblasts by PMA}

It has been shown that the expression of type I collagen was driven by the EGFR signal in fibroblasts. ${ }^{12}$ To determine the activation of EGFR, the primary culture of dermal fibroblasts was treated by PMA, and then the expressions of EGFR and phosphorylated EGFR were examined. As a result, phosphorylated EGFR was increased by the PMA treatment dose-dependently, and the degree was higher in TACE-Tg mice than in WT mice (Figure 7c).

\section{DISCUSSION}

Association of the TACE expression with the pathogenesis of inflammation and fibrosis has been documented in animal models. ${ }^{7-9}$ In humans, TACE has also been demonstrated to be involved in the pathogenesis of inflammatory and fibrous connective tissue diseases, such as rheumatoid arthritis ${ }^{13,14}$ and systemic sclerosis (SSc). ${ }^{15}$ Bohgaki et al ${ }^{15}$ reported that TACE was overexpressed in peripheral blood monocytes of 


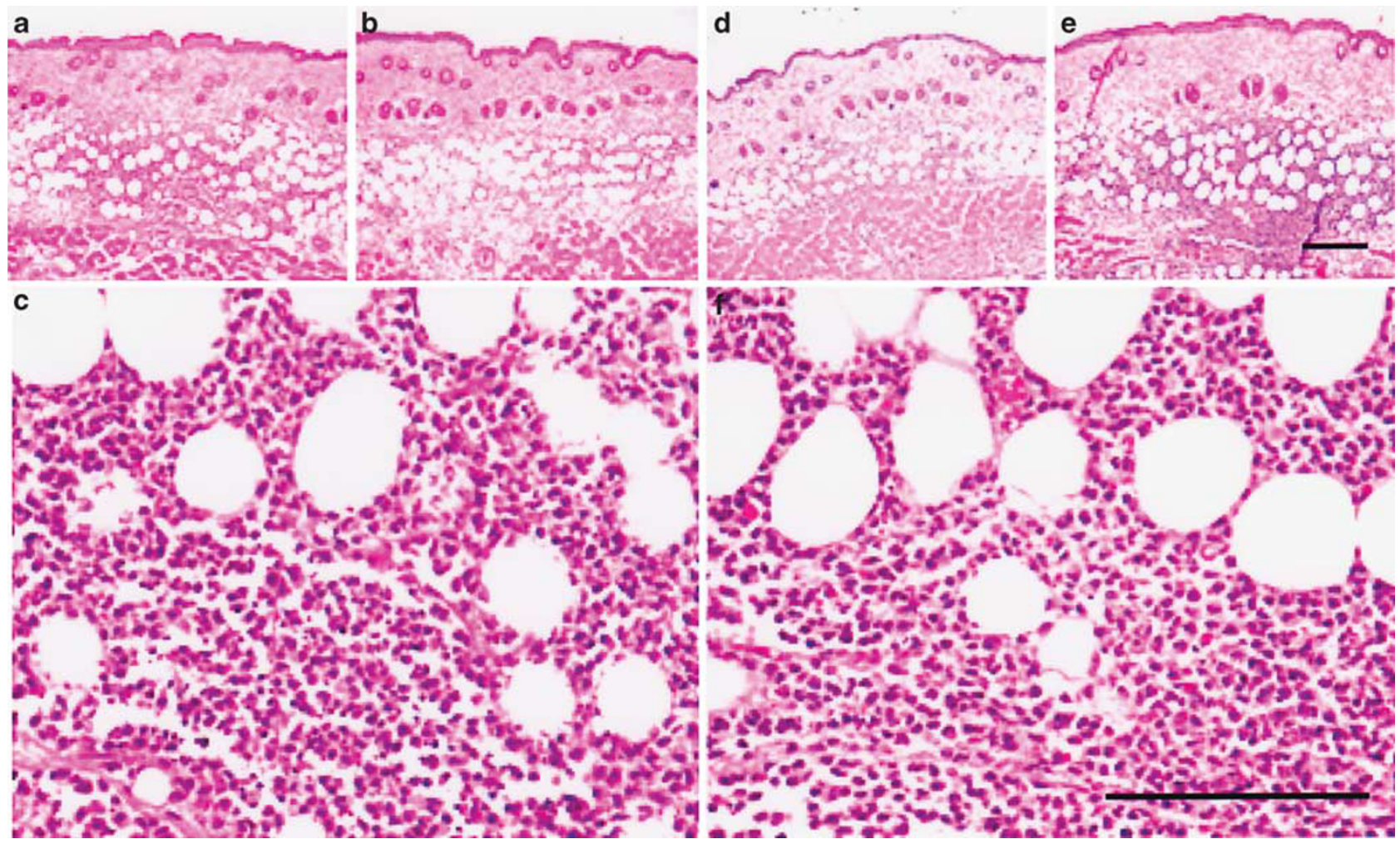

Figure 5 Subcutaneous inflammatory cell infiltration at sites with PMA injection At 1 day after the PMA injection, the sites with PMA injection were excised from TACE-Tg $(n=5)$ and WT $(n=8)$ mice, and then subjected to HE staining (a, b, c: TACE-Tg; d, e, f: WT; bar: $100 \mu \mathrm{M})$. Representative photos are shown.

patients with early stage of SSc, and that the TACE expression was decreased by treatment. These findings suggest that the overexpression of TACE in monocytes might be critically implicated in the development of SSc. On the other hand, it remains elusive how TACE in organ cells can be implicated in the pathogenesis of inflammation and fibrosis.

In the present study, TACE-Tg mice were generated in order to answer the question (Figure 1). As a majority of the transgene-derived TACE were expressed as an inactive form, no spontaneous phenotype occurred in TACE-Tg mice (Supplementary Figure 1). However, furin could convert the transgene-derived inactive TACE to active form; thus, TACE$\mathrm{Tg}$ mice were regarded as inducible models of TACE overexpression and activation (Figure 2). Interestingly, furin failed to activate endogenous TACE unlike the transgenederived TACE. Although the reason should be revealed by further studies, it is possible that undetermined factors in tissue lysates interfered with the measurement of TACE activity when inactive TACE were converted to active form in the tissue lysates. The amount of the putative inhibitory factors seemed to be enough to mask the TACE activity in WT samples mostly, but insufficient to mask that in TACE-Tg samples with overexpression of TACE.

As furin functions exclusively in the cytoplasm in vivo, we employed PMA as stimulant to induce TACE activation in TACE-Tg mice (Figure 3). As a result, the overexpression and activation of TACE in fibroblasts were demonstrated to augment dermal fibrosis after inflammation (Figures 4-6). The subcutaneous injection of PMA into TACE-Tg mice activated TACE in the tissue 1 day later and induced subsequent dermal fibrosis 7 days later. As PMA-induced activation of TACE already returned to the baseline level at day 7 , it remained unclear whether the TACE overexpression and activation at day 1 were critically associated with the increased fibrosis at day 7. Although further studies are needed to clarify the association, it is possible that the TACEdependent type I collagen induction at an early state in inflammation could make an orientation toward subsequent fibrosis. Interestingly, the degree of dermal fibrosis 7 days after PMA injection was significantly higher in TACE-Tg mice than in WT mice, although the degree of inflammatory cell infiltration at day 1 was comparable between the two. These findings suggest that the overexpression of TACE is related to fibrosis after inflammation rather than inflammation itself.

There is a controversy over the contribution of TACE to tissue fibrosis. Terao et $a l^{9}$ demonstrated that TACE contributed to dermal fibrosis using murine bleomycininduced scleroderma model. This finding corresponds to our results. On the contrary, Leco et al ${ }^{16}$ reported that lung emphysema, an opposite phenotype of fibrosis, developed in tissue inhibitor of metalloproteinase 3 (TIMP-3)-deficient 

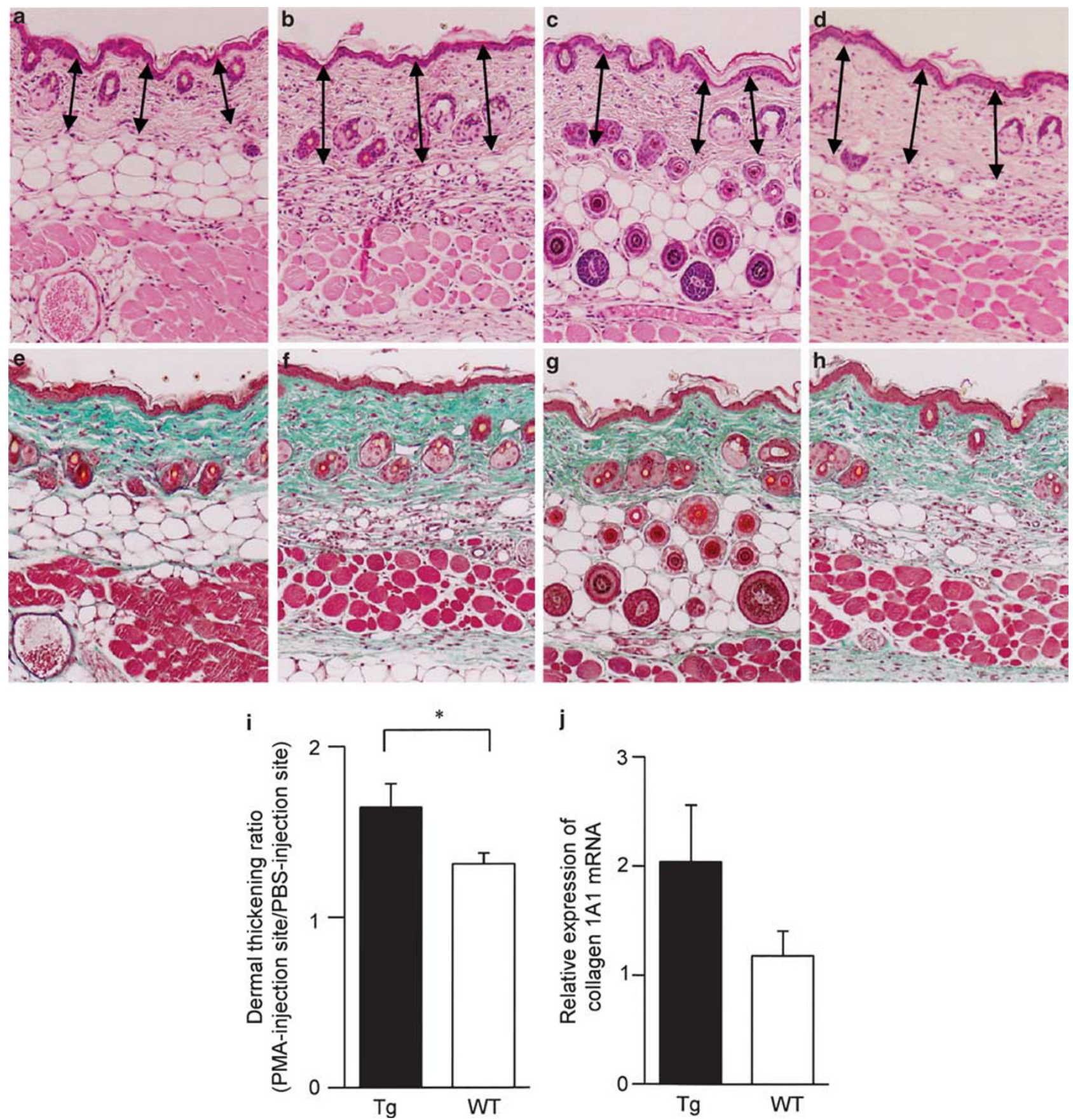

Figure 6 Dermal fibrosis at sites with PMA injection. At 7 days after the PMA injection, the sites with PMA injection (b, d, $\mathbf{f}, \mathbf{h})$ and the sites with PBS injection (a, c, e, g) were excised from TACE-Tg $(n=5)$ and WT $(n=8)$ mice, and then subjected to HE (a-d) and EM (e-h) staining $(\mathbf{a}, \mathbf{b}, \mathbf{e}, \mathbf{f}:$ TACE-Tg, $n=5 ; \mathbf{c}, \mathbf{d}, \mathbf{g}, \mathbf{h}: \mathrm{WT}, n=8$ ). Representative photos are shown. Dermal thickening ratios were calculated as follows: dermal thickness measured at three random points of the sites with PMA injection (arrows in $\mathbf{b}$ and $\mathbf{d}$ )/mean value of dermal thickness measured at three random points of the sites with PBS injection (arrows in $\mathbf{a}$ and $\mathbf{c}$ ), and then were compared between TACE-Tg and WT mice (i). The skin tissues at sites with PMA and PBS injections were obtained at day 7, the expression of type I collagen (collagen 1A1) was examined by real-time RT-PCR, and then fold increase by the PMA injection was compared between TACE-Tg $(n=5)$ and WT $(n=8)$ mice $(j) .{ }^{*} P<0.05$.

mice. As TIMP-3 functions as a TACE inhibitor in vivo, TIMP-3-deficient mice have been documented as a TACE activation model. ${ }^{17,18}$ Therefore, this finding suggests that TACE plays an opposite role in induction of tissue fibrosis and is contradictory to our results. However, TIMP-3- deficient mice are not necessarily an ideal model of TACE activation in vivo because TIMP-3 inhibits not only TACE but also other metalloproteinases.

To confirm the contribution of TACE overexpression in fibroblasts to dermal fibrosis, the expression of type I 

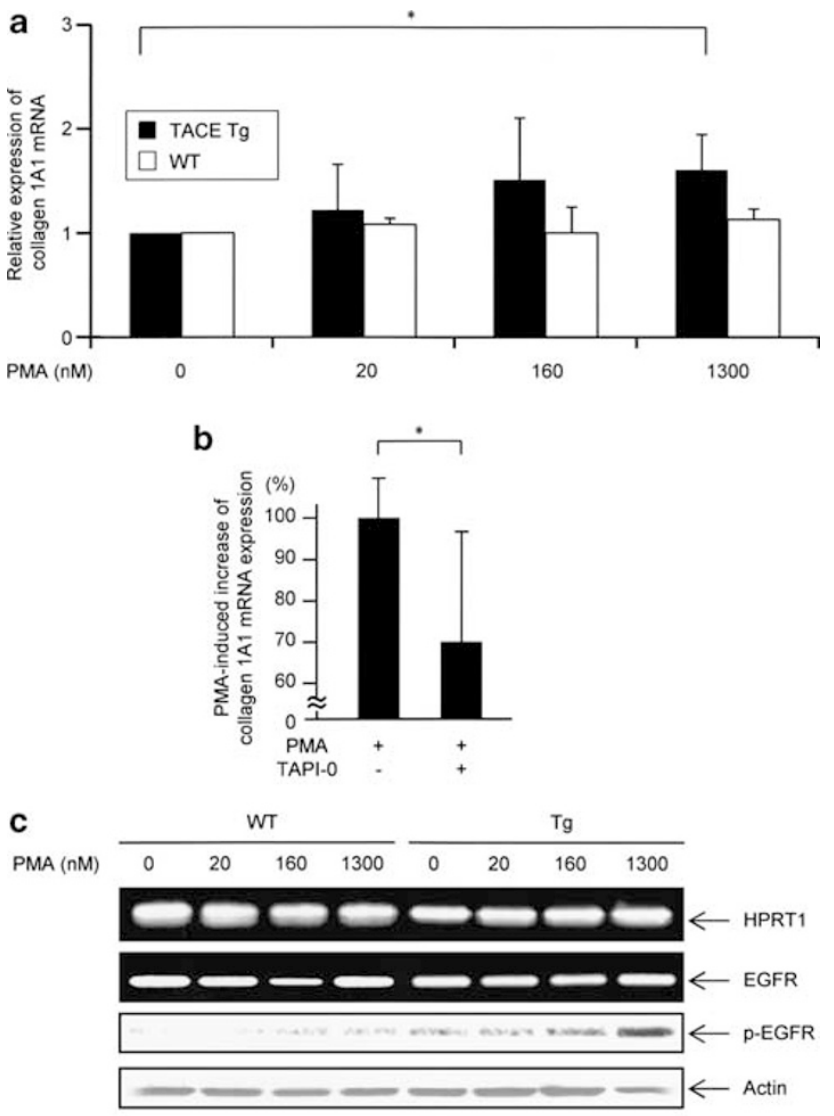

Figure 7 Increased type I collagen and phosphorylated EGFR in fibroblasts by PMA. The primary culture of dermal fibroblasts (TACE-Tg: $n=3$, WT: $n=3$ ) was treated by PMA at respective concentrations of 0 , 20,160 , and $1300 \mathrm{nM}$. After $4 \mathrm{~h}$ of incubation at $37^{\circ} \mathrm{C}$, RNA was extracted from the cells, and then the expression of type I collagen (collagen 1A1) was examined by real-time RT-PCR (a). The fibroblasts derived from TACE-Tg mice were treated by $1300 \mathrm{nM}$ of PMA with or without $25 \mu \mathrm{g} / \mathrm{ml}$ of TAPI- 0 at $37^{\circ} \mathrm{C}$. After $4 \mathrm{~h}$ of incubation, RNA was extracted from the cells, and then the expression of type I collagen (collagen 1A1) was examined by real-time RT-PCR (b). The PMA-induced increase of type I collagen (collagen $1 \mathrm{~A} 1$ ) was set as $100 \% .{ }^{*} P<0.05$. To determine the activation of EGFR, the expressions of EGFR and phosphorylated EGFR were examined (c). The primary culture of dermal fibroblasts was treated by PMA at respective concentrations of $0,20,160$, and $1300 \mathrm{nM}$. After $1 \mathrm{~h}$ of incubation at $37^{\circ} \mathrm{C}$, RNA and cell lysates were extracted. The RNA was served for RT-PCR using the HPRT1 and EGFR primers. The lysates adjusted ranging from 10 to $40 \mu \mathrm{g} /$ lane were fractionated on $7.5 \%$ SDS polyacrylamide gel, and then transferred onto PVDF membranes. After blocking by TBS-T containing $1 \%$ nonfat milk, the membranes were incubated with 1:2500 dilution of the anti-phosphorylated EGFR (p-EGFR) antibody overnight at $4{ }^{\circ} \mathrm{C}$. After 3 times of wash by TBS-T, the membranes were next incubated with 1:25000 dilution of the peroxidase-labeled secondary antibodies overnight at $4{ }^{\circ} \mathrm{C}$. Protein bands were detected using ECL Advance Western Blotting Detection kit. As an internal control, the amount of actin was monitored by the anti-actin antibody. Experiments were repeated 3 times, and similar results were reproduced. Representative data are shown.

collagen using primary culture of dermal fibroblasts was examined in this study. Results indicated that PMA effectively activated TACE and subsequently increased expression of the type I collagen in the fibroblasts derived from TACE-Tg mice (Figure 7a). Furthermore, the induction of type I collagen by PMA was significantly inhibited by the TACE inhibitor (Figure $7 \mathrm{~b}$ ). These findings support our conclusion that TACE overexpression and activation in fibroblasts could contribute to dermal fibrosis. However, this does not necessarily mean that TACE exclusively regulates the PMA-induced type I collagen expression in dermal fibroblasts because TAPI-0 is not a specific inhibitor of TACE.

The substrates of TACE involved in the process of dermal fibrosis after the PMA-induced inflammation have not been identified. However, the amount of phosphorylated EGFR was increased by the PMA treatment of dermal fibroblasts (Figure 7c). As the expression of type I collagen could be driven by the EGFR signal, ${ }^{12}$ TACE activated by PMA could shed off putative EGFR ligands on the surface of fibroblasts. Subsequently, the soluble EGFR ligands could bind and activate EGFR on fibroblasts through the autocrine and paracrine pathways and increase the type I collagen expression resulting in induction of dermal fibrosis. With regard to the EGF signaling pathway, EGFR ligands, including transforming growth factor- $\alpha$ (TGF- $\alpha$ ), heparinbinding EGF (HB-EGF), amphiregulin, and epiregulin, may be the candidates shed by TACE. Among them, amphiregulin is known to be expressed in fibroblasts; ${ }^{19}$ thus, this molecule is the next target in our continuing study.

The EGFR expression has been reported to be upregulated in dermal fibroblasts of SSc patients. ${ }^{20}$ In the present study, the EGFR expression in TACE-Tg-derived dermal fibroblasts did not appear to be increased by PMA in vitro. This could be interpreted by the short duration of PMA stimulation ( $1 \mathrm{~h}$ stimulation) in the experiments. In addition, murine bleomycin-induced lung fibrosis could be suppressed by the EGFR tyrosine kinase inhibitor. ${ }^{21}$ These findings suggest that the EGF signaling pathway comes to a great interest as the mechanism bridging the TACE expression and the pathogenesis of fibrosis.

In summary, the collective findings suggest the possibility that overexpression of TACE in fibroblasts could contribute to the pathogenesis of dermal fibrosis after inflammation. Further studies are needed to reveal the process leading to fibrosis; however, our data suggest that TACE and EGFR on fibroblasts may be novel therapeutic targets of dermal fibrosis, which is induced after diverse inflammatory disorders of the skin.

Supplementary Information accompanies the paper on the Laboratory Investigation website (http://www.laboratoryinvestigation.org)

\section{DISCLOSURE/CONFLICT OF INTEREST}

The authors declare no conflict of interest.

1. Black RA, Rauch CT, Kozlosky CJ, et al. A metalloproteinase disintegrin that releases tumour-necrosis factor- $\alpha$ from cells. Nature 1997;385: 729-733. 
2. Moss $\mathrm{ML}$, Jin $\mathrm{SL}$, Milla $\mathrm{ME}$, et al. Cloning of a disintegrin metalloproteinase that processes precursor tumour-necrosis factor- $\alpha$. Nature 1997;385:733-736.

3. Van Wart HE, Birkedal-Hansen $H$. The cysteine switch: a principle of regulation of metalloproteinase activity with potential applicability to the entire matrix metalloproteinase gene family. Proc Natl Acad Sci USA 1990;87:5578-5582.

4. Grams F, Huber R, Kress LF, et al. Activation of snake venom metalloproteinases by a cysteine switch-like mechanism. FEBS Let 1993;335:76-80.

5. Kang T, Zhao YG, Pei D, et al. Intracellular activation of human adamalysin 19/disintegrin and metalloproteinase 19 by furin occurs via one of the two consecutive recognition sites. J Biol Chem 2002;277:25583-25591.

6. Endres K, Anders A, Kojro E, et al. Tumor necrosis factor- $\alpha$ converting enzyme is processed by proprotein-convertases to its mature form which is degraded upon phorbol ester stimulation. Eur J Biochem 2003:270:2386-2393.

7. Doggrell SA. TACE inhibition: a new approach to treating inflammation. Expert Opin Investig Drugs 2002;11:1003-1006.

8. Shimizu M, Hasegawa N, Nishimura T, et al. Effects of TNF- $\alpha$-converting enzyme inhibition on acute lung injury induced by endotoxin in the rat. Shock 2009;32:535-540.

9. Terao $M$, Murota $H$, Kitaba $S$, et al. Tumor necrosis factor- $\alpha$ processing inhibitor-1 inhibits skin fibrosis in a bleomycin-induced murine mode of scleroderma. Exp Dermatol 2010;19:38-43.

10. Mohler KM, Sleath PR, Fitzner JN, et al. Protection against a lethal dose of endotoxin by an inhibitor of tumour necrosis factor processing Nature 1994;370:218-220.

11. Maltzan K, Tan W, Pruett SB. Investigation of the role of TNF- $\alpha$ converting enzyme (TACE) in the inhibition of cell surface and soluble TNF- $\alpha$ production by acute ethanol exposure. PLoS One 2012;7:e29890.
12. Wu D, Peng F, Zhang B. Collagen I induction by high glucose levels is mediated by epidermal growth factor receptor and phosphoinositide 3-kinase/Akt signalling in mesangial cells. Diabetologia 2007;50: 2008-2018.

13. Ohta $S$, Harigai $M$, Tanaka $M$, et al. Tumor necrosis factor- $\alpha(T N F-\alpha)$ converting enzyme contributes to production of TNF- $\alpha$ in synovial tissues from patients with rheumatoid arthritis. J Rheumatol 2001;28:1756-1763.

14. Moss ML, Sklair-Tavron L, Nudelman R. Drug insight: tumor necrosis factor-converting enzyme as a pharmaceutical target for rheumatoid arthritis. Nat Clin Pract Rheumatol 2008;4:300-309.

15. Bohgaki T, Amasaki $Y$, Nishimura N, et al. Up regulated expression of tumour necrosis factor $\alpha$ converting enzyme in peripheral monocytes of patients with early systemic sclerosis. Ann Rheum Dis 2005;64:1165-1173.

16. Leco KJ, Waterhouse $\mathrm{P}$, Sanchez $\mathrm{OH}$, et al. Spontaneous air space enlargement in the lungs of mice lacking tissue inhibitor of metalloproteinases-3 (TIMP-3). J Clin Invest 2001;108:817-829.

17. Mohammed FF, Smookler DS, Taylor SE, et al. Abnormal TNF activity in $\mathrm{Timp}^{-/-}$mice leads to chronic hepatic inflammation and failure of liver regeneration. Nat Genet 2004;36:969-977.

18. Smookler DS, Mohammed FF, Kassiri Z, et al. Tissue inhibitor of metalloproteinase 3 regulates TNF-dependent systemic inflammation. J Immunol 2006;176:721-725.

19. Torring N, Sorensen BS, Bosch ST, et al. Amphiregulin is expressed in primary cultures of prostate myofibroblasts, fibroblasts, epithelial cells, and in prostate tissue. Prostate Cancer Prostatic Dis 1998;1:262-267.

20. Tokiyama K, Yokota E, Niho Y. Epidermal growth factor receptor of fibroblasts from patients with scleroderma. J Rheumatol 1990;17: 1463-1468.

21. Wang $P$, Tian Q, Liang ZX, et al. Gefitinib attenuates murine pulmonary fibrosis induced by bleomycin. Chin Med J (Engl) 2010;123:2259-2264. 